CASE REPORT

\title{
Tubular aggregate myopathy presenting with acute type II respiratory failure and severe orthopnoea
}

\author{
N Shahrizaila, W S Lim, D K Robson, W J Kinnear, A J Wills
}

Thorax 2006;61:89-90. doi: 10.1136/thx.2004.028233

Acute hypercapnic respiratory failure (AHRF) is a common reason for hospital admission. Most patients have an underlying chronic lung disease such as chronic obstructive pulmonary disease. We report the case of a man who presented with AHRF secondary to tubular aggregate myopathy.

A 56 year old man presented with a 2 month history of breathlessness, orthopnoea, and decreased exercise tolerance. Further enquiry revealed a several month history of pedal oedema. He was an ex-smoker of 25 years. There was no significant family history.

\section{Initial findings and management}

On examination his respiratory rate was 18 breaths/min and his jugular venous pressure was raised to $5 \mathrm{~mm} \mathrm{Hg}$ at $45^{\circ}$ incline. Auscultation revealed reduced breath sounds and a pan systolic murmur at the left sternal edge. There was pitting oedema to his knees. Arterial blood gas tensions on air were as follows: $\mathrm{pH} 7.38, \mathrm{PaO}_{2} 6.11 \mathrm{kPa}, \mathrm{PaCO}_{2} 8.99 \mathrm{kPa}$, $\mathrm{HCO}_{3} 38.9 \mathrm{mmol} / \mathrm{l}$, oxygen saturation $79 \%$. A chest radiograph revealed poorly expanded lungs. Laboratory investigations showed polycythaemia with a haemoglobin level of $19.0 \mathrm{~g} / \mathrm{dl}$ and haematocrit of 0.634 (normal range 0.3-0.54).

A diagnosis of an acute exacerbation of previously undiagnosed COPD was made by the admitting doctors and the patient was treated with bronchodilators and controlled oxygen therapy. However, his condition deteriorated with worsening hypercapnia. Senior advice regarding the decision to commence non-invasive positive pressure ventilation (NIV) was sought.

\section{Further findings and management}

Upon senior review, abdominal paradox indicating diaphragmatic weakness was noted. The rest of his neurological examination was normal. Spirometric tests revealed a forced expiratory volume in 1 second $\left(\mathrm{FEV}_{1}\right)$ of $1.25 \mathrm{l}$ and forced vital capacity (FVC) of $1.55 \mathrm{l}$, both $35 \%$ predicted for his age and height. Subsequent respiratory muscle testing revealed expiratory mouth pressure of $13 \mathrm{kPa}$ (normal $>9 \mathrm{kPa}$ ), inspiratory mouth pressure of $2.8 \mathrm{kPa}$ (normal $>6 \mathrm{kPa}$ ) and sniff nasal pressure of $3 \mathrm{kPa}$ (normal $>8 \mathrm{kPa}$ ). On lying from a sitting position there was a $20 \%$ fall in his FVC (normal range $<15 \%$ ). A diagnosis of bilateral diaphragmatic weakness was made and, following initiation of NIV, his condition improved.

Further investigations included creatine kinase, vacuolated lymphocytes, acetylcholine receptor antibodies, and $\alpha_{1}$ antitrypsin levels, all of which were normal. Echocardiography confirmed a dilated right heart with tricuspid regurgitation and globally impaired right ventricle. Quadriceps muscle biopsy revealed many fibres with inclusions which were highlighted by NADH-Tr and Gomori trichrome preparations. Electron microscopic examination showed the inclusions to be tubular aggregates (fig l).

He was discharged 13 days later on overnight NIV alone. At discharge his arterial blood gas tensions were: $\mathrm{pH}$ 7.39, $\mathrm{PaO}_{2} 9.44 \mathrm{kPa}, \mathrm{PaCO}_{2} 7.75 \mathrm{kPa}, \mathrm{HCO}_{3} 31.2 \mathrm{mmol} / \mathrm{l}$, oxygen saturation $93 \%$. At follow up 2 weeks after discharge there was further improvement in his condition. His quality of sleep had improved and the signs and symptoms of right heart failure had resolved. One year later he remained on overnight NIV with daytime oxygen saturation of $96 \%$ on air and a marked improvement in his spirometric values $\left(\mathrm{FEV}_{1}\right.$ $2.19 \mathrm{l}$ (63\% predicted) and FVC $3.14 \mathrm{l}$ (71\% predicted)). The diaphragmatic weakness persisted with a $32 \%$ fall in supine FVC, inspiratory mouth pressure of $2 \mathrm{kPa}$, and sniff nasal pressure of $2.3 \mathrm{kPa}$.

\section{DISCUSSION}

Acute type II respiratory failure is a common reason for emergency hospital admission. It is most commonly due to an acute exacerbation of COPD. Hypercapnic respiratory failure resulting from hypoventilation is uncommon, especially in previously fit individuals. Ventilation is compromised when there is inadequate central nervous system drive as well as reduced capacity of the respiratory muscles to manage the load imposed on them. ${ }^{1}$

Our patient presented with severe orthopnoea. Clinically, he was in right heart failure and further examination revealed abdominal paradox. Investigations highlighted type II respiratory failure, reduced lung volumes, inspiratory muscle weakness, and right ventricular failure. All these findings pointed towards a diagnosis of diaphragmatic weakness leading to hypoventilation as the cause of acute hypercapnic respiratory failure (AHRF). Muscle biopsy confirmed tubular aggregate myopathy.

Tubular aggregates in skeletal muscles are derived from sarcoplasmic reticulum and are thought to be an adaptive compensatory response to increased intracellular calcium levels which would have caused irreversible contraction of muscle fibres. ${ }^{23}$ The reported clinical presentation of tubular aggregate myopathy typically takes the form of myalgia, cramps, or limb muscle weakness. ${ }^{45}$ Our personal observation of other cases with tubular aggregate myopathy have been consistent with this. To our knowledge, bilateral diaphragmatic weakness as a presenting feature of tubular aggregate myopathy in the absence of limb weakness has not previously been reported.

Other neuromuscular diseases reported to present as AHRF include amyotrophic lateral sclerosis, myotonic dystrophy, and acid maltase deficiency. In these conditions, respiratory failure was attributed to respiratory muscle weakness. ${ }^{6-8}$

Abbreviations: AHRF, acute hypercapnic respiratory failure; COPD, chronic obstructive pulmonary disease; $\mathrm{FEV}_{1}$, forced expiratory volume in 1 second; FVC, forced vital capacity; NIV, non-invasive ventilation 


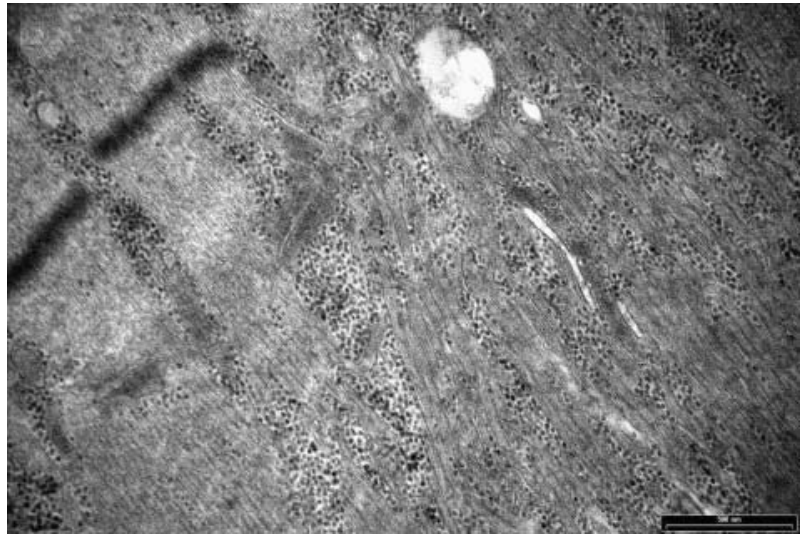

Figure 1 Electron micrograph of muscle biopsy specimen showing tubular aggregates (bar $=500 \mathrm{~nm}$ ).

The clinical combination of severe orthopnoea associated with abdominal paradox was key to the correct diagnosis in this case. Although patients presenting with AHRF due to neuromuscular disease comprise only a small group, they may benefit from assisted ventilation, not only in the acute setting but also in the longer term. This contrasts with the longer term prognosis of patients with COPD and type II respiratory failure requiring NIV. ${ }^{9}$ Early and accurate diagnosis in these patients is therefore essential.

\section{Authors' affiliations}

N Shahrizaila, A J Wills, Department of Neurology, Queen's Medical Centre, Nottingham, UK

W S Lim, Department of Respiratory Medicine, Nottingham City Hospital, Nottingham, UK
D K Robson, Department of Histopathology, Queen's Medical Centre, Nottingham, UK

W J Kinnear, Department of Respiratory Medicine, Queen's Medical Centre, Nottingham, UK

Competing interests: none declared.

Correspondence to: Dr N Shahrizaila, Department of Neurology, Queen's Medical Centre, Nottingham NG7 1UH, UK; tshahrizaila@ hotmail.com

Received 18 May 2004

Accepted 13 August 2004

\section{REFERENCES}

1 Goldstone JC, Green M, Moxham J. Maximum relaxation rate of the diaphragm during weaning from mechanical ventilation. Thorax 1994:49:54-60.

2 Bendahan D, Pouget J, Pellissier JF, et al. Magnetic resonance spectroscopy and histological study of tubular aggregates in a familial myopathy. I Neurol Sci 1996;139:149-55

3 Martin JJ, Ceuterick C, Van Goethem G. On a dominantly inherited myopathy with tubular aggregates. Neuromuscul Disord 1997;7:512-20.

4 Morgan-Hughes JA. Tubular aggregates in skeletal muscle: their functional significance and mechanisms of pathogenesis. Curr Opin Neurol 1998; 11:439-42.

5 Jacques TS, Holton J, Watts PM, et al. Tubular aggregate myopathy with abnormal pupils and skeletal deformities. J Neurol Neurosurg Psychiatry 2002;73:324-6.

6 Parhad IM, Clark AW, Barron KD, Staunton SB. Diaphragmatic paralysis in motor neuron disease. Report of two cases and a review of the literature. Neurology 1978;28:18-22.

7 Rosenow EC 3rd, Engel AG. cid maltase deficiency in adults presenting as respiratory failure. Am J Med 1978;64:485-91.

8 Begin P, Mathieu J, Almirall J, et al. Relationship between chronic hypercapnia and inspiratory-muscle weakness in myotonic dystrophy. Am J Respir Crit Care Med 1997;156:133-9.

9 Wijkstra PJ, Lacasse Y, Guyatt GH, et al. Nocturnal non-invasive positive pressure ventilation for stable chronic obstructive pulmonary disease. Cochrane Database Syst Rev 2002;(3):CD002878. 